\title{
Arterial Spin-Labeling Parameters Influence Signal Variability and Estimated Regional Relative Cerebral Blood Flow in Normal Aging and Mild Cognitive Impairment: FAIR versus PICORE Techniques
}

\author{
K.-O. Lövblad, M.-L. Montandon, M. Viallon, C. Rodriguez, S. Toma, X. Golay, P. Giannakopoulos, and S. Haller
}

\begin{abstract}
BACKGROUND AND PURPOSE: Arterial spin-labeling is a noninvasive method to map cerebral blood flow, which might be useful for early diagnosis of neurodegenerative diseases. We directly compared 2 arterial spin-labeling techniques in healthy elderly controls and individuals with mild cognitive impairment.
\end{abstract}

MATERIALS AND METHODS: This prospective study was approved by the local ethics committee and included 198 consecutive healthy controls (mean age, $73.65 \pm 4.02$ years) and 43 subjects with mild cognitive impairment (mean age, $73.38 \pm 5.85$ years). Two pulsed arterial spin-labeling sequences were performed at 3T: proximal inversion with a control for off-resonance effects (PICORE) and flow-sensitive alternating inversion recovery technique (FAIR). Relative cerebral blood flow maps were calculated by using commercial software and standard parameters. Data analysis included spatial normalization of gray matter-corrected relative CBF maps, whole-brain average, and voxelwise comparison of both arterial spin-labeling sequences.

RESULTS: Overall, FAIR yielded higher relative CBF values compared with PICORE (controls, $32.7 \pm 7.1$ versus $30.0 \pm 13.1 \mathrm{~mL} / \mathrm{min} / 100 \mathrm{~g}, P=$ .05 ; mild cognitive impairment, $29.8 \pm 5.4$ versus $26.2 \pm 8.6 \mathrm{~mL} / \mathrm{min} / 100 \mathrm{~g}, P<.05$; all, $32.2 \pm 6.8$ versus $29.3 \pm 12.3 \mathrm{~mL} / \mathrm{min} / 100 \mathrm{~g}, P<.05$ ). FAIR had lower variability (controls, $36.2 \%$ versus $68.8 \%, P<.00001$; mild cognitive impairment, $18.9 \%$ versus $22.9 \%, P<.0001$; all, $34.4 \%$ versus $64.9 \% P<.00001$ ). The detailed voxelwise analysis revealed a higher signal for FAIR, notably in both convexities, while PICORE had higher signal predominantly in deep cerebral regions.

CONCLUSIONS: Overall, FAIR had higher estimated relative CBF and lower interindividual variability than PICORE. In more detail, there were regional differences between both arterial spin-labeling sequences. In summary, these results highlight the need to calibrate arterial spin-labeling sequences.

ABBREVIATIONS: ASL = arterial spin-labeling; FAIR = flow-sensitive alternating inversion recovery technique; $\mathrm{MCI}=$ mild cognitive impairment; $\mathrm{PICORE}=$ proximal inversion with a control for off-resonance effects; $r \mathrm{CBF}=$ relative cerebral blood flow

A rterial spin-labeling (ASL) is a noninvasive perfusion technique that uses the labeled spins in arterial water as an endogenous tracer. In principle, 2 images are acquired, 1 control image with no blood labeling and 1 tag image in which arterial blood is labeled. The technique was developed 2 decades ago ${ }^{1}$ and is feasible on $1.5 \mathrm{~T}$ scanners but became increasingly used with the introduction of clinical 3T scanners. Recent investigations high-

Received November 17, 2014; accepted after revision December 5. From the Divisions of Diagnostic and Interventional Neuroradiology (K.-O.L., M.-L.M., M.V., S.H.) and Psychiatry (C.R., S.T., P.G.), Geneva University Hospitals, Geneva, Switzerland; CREATIS (M.V.), UMR CNRS 5220-Institut National de la Santé et de la Recherche Médicale U1044, INSA de Lyon, Université de Lyon, Centre Hospitalier Universitaire de Saint Etienne, Saint Etienne, France; and Institute of Neurology (X.G.), University College London, London, United Kingdom.

This work was supported by Swiss National Foundation grant SNF 3200B0-116193 and SPUM 33CM30-124111. Collaboration with X.G. was supported by COST Action BM1103 on "ASL in Dementia" and by the National Institute for Health Research University College London Hospitals Biomedical Research Centre. light the utility of ASL in the domain of neurodegeneration, notably mild cognitive impairment (MCI) and Alzheimer disease, ${ }^{2-5}$ for review. ${ }^{6}$ More recently, ASL was even able to predict very early cognitive decline in healthy elderly controls (ie, the earliest stage of neurodegeneration). ${ }^{7}$ These investigations demonstrated consistent reduction in relative cerebral blood flow ( $\mathrm{rCBF}$ ), notably in the posterior cingulate cortex, which overlaps the patterns of hypometabolism on $\left[{ }^{18} \mathrm{~F}\right] \mathrm{FDG}-\mathrm{PET}$ in clinically overt and incipient cases of Alzheimer disease. ${ }^{8-11}$ The correspondence between ASL and PET findings is consistent with the known perfusion-metabolism coupling in the brain. ${ }^{12,13}$

Please address correspondence to Sven Haller, MD, Service Neuro-Diagnostique et Neuro-Interventionnel DISIM, University Hospitals of Geneva, Rue Gabrielle Perret-Gentil 4, 1211 Geneva 14, Switzerland; e-mail: sven.haller@hcuge.ch

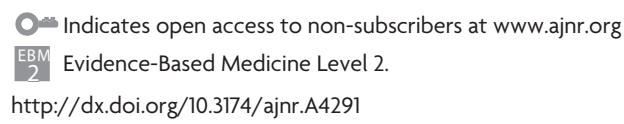


Summary of the essential imaging parameters for PICORE and FAIR ASL sequences

\begin{tabular}{|c|c|c|}
\hline & PICORE Q2TIPS & FAIR-QUIPSS II \\
\hline Matrix & $64 \times 64$ & $64 \times 64$ \\
\hline No. of sections & 20 & 24 \\
\hline Pixel size $\left(\mathrm{mm}^{2}\right)$ & $3.44 \times 3.44$ & $3.44 \times 3.44$ \\
\hline Section thickness (mm) & 6 & 4 \\
\hline Gap (mm) & 1 & 0 \\
\hline FOV & 220 & 220 \\
\hline Parallel imaging (GRAPPA) & 2 & 2 \\
\hline Phase, partial Fourier & $7 / 8$ & $6 / 8$ \\
\hline Section order & Ascending & Ascending \\
\hline Section orientation & ACPC aligned & ACPC aligned \\
\hline Fat saturation & Yes & Yes \\
\hline Coil-combination algo & Sum of squares & Sum of squares \\
\hline Bandwidth & $2604 \mathrm{~Hz} / \mathrm{Px}$ & $2368 \mathrm{~Hz} / \mathrm{Px}$ \\
\hline Echo-spacing & 0.47 & 0.49 \\
\hline Saturation slab & $\begin{array}{l}\text { Parallel feet, gap }=25.6 \mathrm{~mm} \text {, } \\
\text { thickness }=110 \mathrm{~mm}\end{array}$ & $\begin{array}{l}\text { Parallel feet/head gap }=10 \mathrm{~mm} \text {, } \\
\text { thickness }=100 \mathrm{~mm}\end{array}$ \\
\hline TE (ms) & 21 & 12 \\
\hline TR (ms) & 5000 & 4000 \\
\hline TI 1 (ms) & 700 & 600 \\
\hline TI 2 (ms) & 1800 & 1600 \\
\hline Saturation stop time (ms) & 1600 & \\
\hline Flow limit (cm/s) & 5 & 100 \\
\hline No. of images & 71 & 105 \\
\hline Total acquisition time & 6 Minutes 12 seconds & 7 Minutes 6 seconds \\
\hline Mo reference image & No & Yes \\
\hline
\end{tabular}

Note:-ACPC indicates anterior/posterior commissure; algo, algorithm; Q2TIPS, quantitative imaging of perfusion with a single subtraction with thin-section $\mathrm{TI}_{1}$ periodic saturation; QUIPSS II, quantitative imaging of perfusion using a single subtraction II; Mo, steady-state magnetization; GRAPPA, generalized autocalibrating partially parallel acquisitions.

\section{MR Imaging}

Imaging data were acquired on a clinical routine whole-body $3 \mathrm{~T}$ MR imaging scanner (Magnetom Trio; Siemens, Erlangen, Germany). Two pulsed arterial spin-labeling approaches were performed for comparison: PICORE $^{15}$ quantitative imaging of perfusion with a single subtraction with thin-section $\mathrm{TI}_{1}$ periodic saturation (Q2TIPS) labeling scheme $^{18}$ and FAIR ${ }^{14}$ combined with quantitative imaging of perfusion using a single subtraction II (QUIPSS II). ${ }^{19}$

The essential parameters for both ASL sequences are illustrated in the Table.

A structural 3D T1 sequence was obtained for spatial normalization $(256 \times$ 256 matrix; 176 sections, $1 \times 1 \times 1 \mathrm{~mm}^{3}$; TE, $2.3 \mathrm{~ms}$; TR, $2300 \mathrm{~ms}$ ). Additional sequences (T2-weighted, diffusion tensor imaging, fluid-attenuated inversion recovery) were acquired and analyzed to rule out concomitant diseases such as ischemic stroke and subdural hematomas and susceptibility artifacts from prior hemorrhage or space-occupying lesions. White

In the context of neurocognitive decline, disease-related alterations in brain perfusion are a priori small, in particular in early stages of the disease. This feature implies that the optimal ASL sequence for the early detection of neurodegenerative processes should have a strong signal and, in particular, a low variability. Unfortunately, ASL has low intrinsic signal and contrast. Consequently, several techniques have been proposed to improve spinlabeling, thereby resulting in a higher signal, including the flowsensitive alternating inversion recovery technique (FAIR) ${ }^{14}$ and the proximal inversion with a control for off-resonance effects (PICORE). ${ }^{15}$

In this prospective investigation, we directly compared 2 pulsed ASL sequences (PICORE versus FAIR) in 241 consecutive elderly controls and MCI participants. Both ASL sequences are in agreement with the recent recommendations on ASL imaging. ${ }^{16}$ We assessed whole-brain average and local signal strength and interindividual variability.

\section{MATERIALS AND METHODS Participants}

The local ethics committee approved this prospective study, and all participants gave informed written consent before inclusion. A total of 241 consecutive subjects who had both PICORE and FAIR ASL sequences were included in this study from an ongoing cohort study with extensive neuropsychological evaluation and multiparametric MR imaging as described in detail before. ${ }^{7}$ The final sample consisted of 198 healthy controls (mean age, $73.65 \pm$ 4.02 years; 116 women; Mini-Mental State Examination score, ${ }^{17}$ $28.36 \pm 1.41$ ) and 43 subjects with MCI (mean age, $73.38 \pm 5.85$ years; 15 women; Mini-Mental State Examination score, $26.93 \pm$ 2.36). matter lesions were analyzed according to the Fazekas score. ${ }^{20}$ Mildto-moderate white matter lesions (Fazekas 1-2) are very common in this age group and were not exclusion criteria. In contrast, participants with severe white matter lesion load (Fazekas 3) were excluded.

\section{Statistical Analysis}

The relative $\mathrm{CBF}$ values were calculated inline on the MR imaging scanner according to Luh et $\mathrm{al}^{18}$ and Wang et al ${ }^{19}$ and under the assumption of constant standard values for hematocrit, $\mathrm{T} 1, \lambda$, and inversion efficiency.

These rCBF maps were further processed with the fMRI of the Brain Software Library (FSL, Version 5.0.2.1; http://www.fmrib. ox.ac.uk/fsl) by using the following data-processing steps: masking of nonbrain voxels by using the Brain Extraction Tool (http://fsl.fmrib.ox.ac.uk/fsl/fslwiki/BET, part of FSL), linear registration of the ASL raw data to the same subject's 3D T1 data by using the FMRIB Linear Image Registration Tool (FLIRT; http:// www.fmrib.ox.ac.uk/), nonlinear spatial registration of the highresolution 3D T1 image to Montreal Neurological Institute standard space (the FMRIB Nonlinear Registration Tool, [FNIRT, http://fsl.fmrib.ox.ac.uk/fsl/fslwiki/FNIRT]), and application of this nonlinear transformation matrix to the ASL rCBF maps to spatially normalize the rCBF maps into Montreal Neurological Institute standard space. ASL rCBF is higher in gray matter than in white matter. To compensate for partial volume effects of the rCBF maps, we calculated gray matter-corrected rCBF maps as reported previously. ${ }^{4,5}$ In principle, the individual 3D T1 images were preprocessed by using FSL voxel-based morphometry to create individual gray matter maps, which were then used to mask the individual ASL rCBF maps to obtain individual gray mattercorrected rCBF maps. 

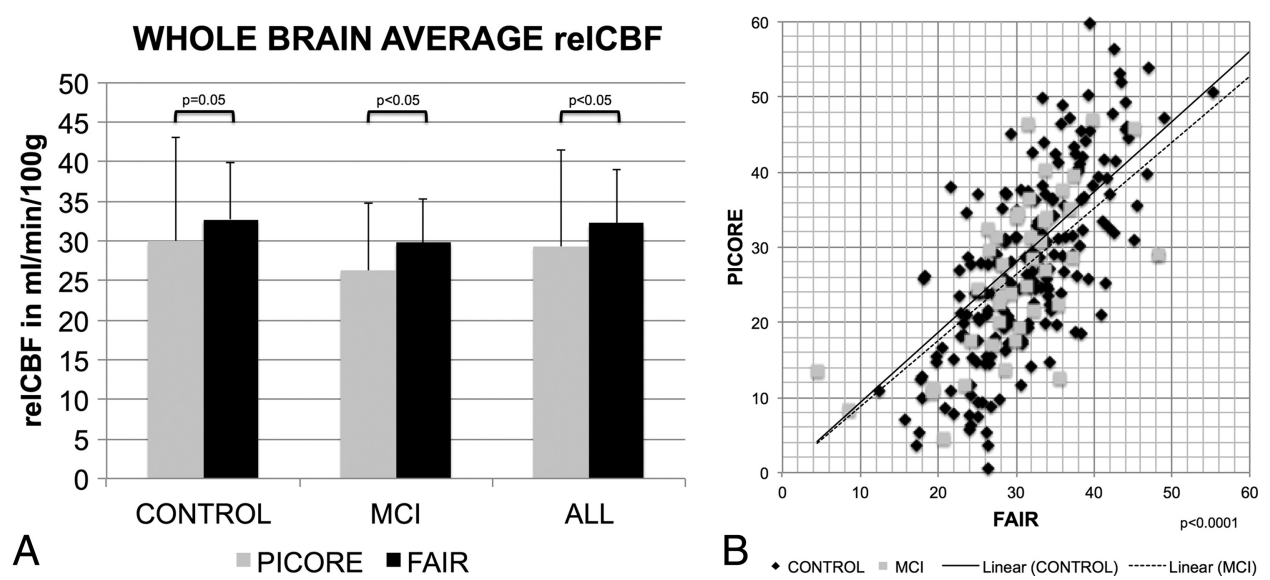

FIG 1. A, The whole-brain average rCBF (relCBF) values in milliliters/minute/100 $\mathrm{g}$ for the $2 \mathrm{ASL}$ sequences, PICORE and FAIR, only for elderly control participants (CONTROL), only for $\mathrm{MCl}$, and for ALL participants. FAIR provided significantly higher signal than $\mathrm{PICORE}(P<.05)$. The error bars indicate SD. B, The correlation of the whole-brain average rCBF between PICORE and FAIR was significant at $P<.0001$. The lines represent the linear fit for CONTROL and $\mathrm{MCl}$ with an intercept of zero.

First, we compared the whole-brain average rCBF values between the 2 ASL techniques by using paired $t$ tests. Additionally, we calculated the Pearson correlation coefficient for the rCBF of both sequences.

Second, we calculated the whole-brain average relative $\mathrm{SD}$ as (Individual rCBF - Group Average rCBF)/Group Average rCBF. These relative SD values were compared between the 2 ASL techniques by using paired $t$ tests.

Third, we applied a voxelwise permutation-based testing (Randomize, part of FSL; http://fsl.fmrib.ox.ac.uk/fsl/fslwiki/ randomise), with threshold-free cluster-enhancement correction for multiple comparisons, ${ }^{21}$ considering fully corrected $P<.05$ as significant.

All analyses were performed 3 times: only for the 198 control participants, only for the 43 subjects with MCI, and for all 241 participants.

\section{RESULTS}

\section{Whole-Brain Average rCBF}

On average across the entire brain (ie, including gray and white matter), FAIR provided higher rCBF values compared with PICORE: controls, $32.7 \pm 7.1$ versus $30.0 \pm 13.1 \mathrm{~mL} / \mathrm{min} / 100 \mathrm{~g}$, $P=.05 ; \mathrm{MCI}, 29.8 \pm 5.4$ versus $26.2 \pm 8.6 \mathrm{~mL} / \mathrm{min} / 100 \mathrm{~g}, P<.05$; all, $32.2 \pm 6.8$ versus $29.3 \pm 12.3 \mathrm{~mL} / \mathrm{min} / 100 \mathrm{~g}, P<.05$ (Fig 1 ). The Pearson correlation for FAIR and PICORE was significant at $P<.0001$ for controls, MCI, and all. The slope of the correlation for all cases was $0.93 \pm 0.03$ (95\% confidence interval, $0.86-0.99)$.

\section{Interindividual Variability in $\mathrm{rCBF}$}

The interindividual variability in $\mathrm{rCBF}$ was estimated as relative to the deviation of the individual whole-brain average $\mathrm{rCBF}$ with respect to the corresponding group mean. FAIR had lower variability than PICORE: controls, $36.2 \%$ versus $68.8 \%, P<.00001$; MCI, $18.9 \%$ versus $22.9 \%, P<.0001$; all, $34.4 \%$ versus $64.9 \% P<.00001$ (Fig 2).

\section{Voxelwise Comparison between FAIR and PICORE}

The average voxelwise rCBF values for FAIR, PICORE, and the difference between both sequences are illustrated in Fig 3.

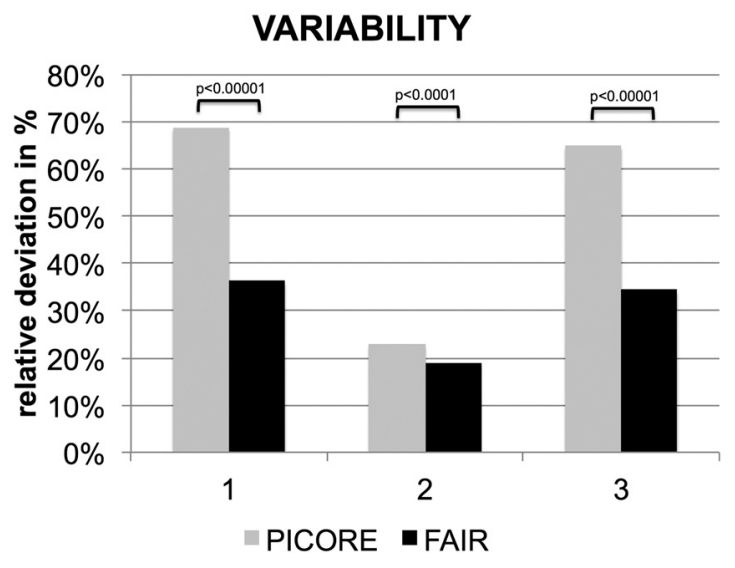

FIG 2. Significantly lower variability of the whole-brain average estimated rCBF for FAIR versus PICORE.

The direct voxelwise comparison between FAIR and PICORE revealed higher rCBF for FAIR in most brain areas, mainly the convexities, which partly corresponded to the anterior circulation. In contrast, PICORE provided higher signal compared with FAIR in the bilateral thalamus and occipital and infratentorial regions, largely but not completely respecting the posterior circulation. Exceptions for these vascular territories are the higher signal for PICORE in the bilateral head of caudate nucleus (lenticulostriate arteries, anterior circulation) and the posterior insula (middle cerebral artery, anterior circulation), while FAIR provided higher signal in the occipital pole (posterior cerebral artery territory) and posterior division of the cingulate gyrus (posterior cerebral artery, posterior circulation) (Fig 4).

\section{DISCUSSION}

Arterial spin-labeling is a promising neuroimaging technique for examining cognitive decline due to the close link between brain perfusion and brain metabolism. ${ }^{12,13}$ Accordingly, the patterns of hypoperfusion in $\mathrm{ASL}^{2-5}$ closely resemble hypometabolism in FDG$\mathrm{PET}^{8-11}$ in MCI and Alzheimer disease. ${ }^{6}$ Because disease-related alterations in brain perfusion in neurodegenerative diseases are a pri- 


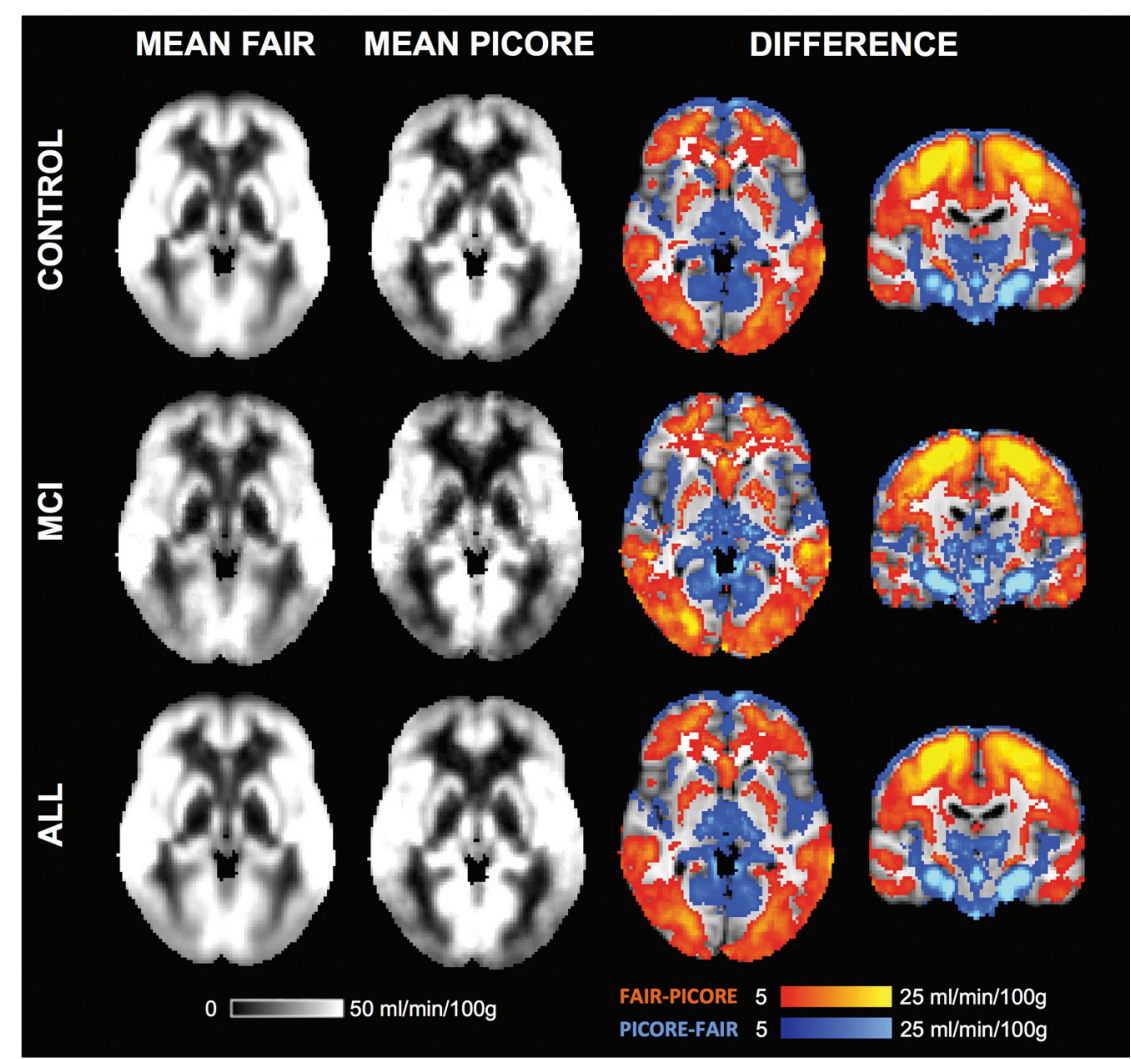

FIG 3. The average $\mathrm{rCBF}$ for FAIR, PICORE, and the difference between both ASL sequences.

our study, the imaging slab was positioned by using the same orientation, and the labeling slab was always parallel to the imaging slab.

Respecting strict standardized procedures, we found that the reproducibility of the same ASL sequence both within and between sessions was fairly good. ${ }^{28}$ Note that both ASL sequences in the current investigation are in agreement with recent recommendations ${ }^{16}$ and were performed within the same session. In the clinical applications of ASL, the sequence-related offset is likely even higher, considering additionally confounding factors such as different MR imaging vendors, machines, and head coils. In summary, these observations highlight the need to calibrate individual ASL sequences, for example, by using flow phantoms to detect subtle perfusion alterations related to neurodegeneration.

Second, when we assessed the regional differences between both ASL sequences in detail, FAIR had higher rCBF in both cerebral convexities, notably in the anterior circulation. In contrast, PICORE had higher values in deep, occipital, and infratentorial regions, largely respecting the

ori small, notably at early stages of the disease, the implemented ASL sequence should have high signal and high reproducibility.

The direct comparison of 2 ASL sequences, which were both implemented with parameters in the range of recent guidelines, ${ }^{16}$ demonstrates that on average across the entire brain, FAIR had significantly lower variability compared with PICORE pulsed ASL, most probably due to the larger number of repetitions. Independent from any ASL pulse sequence, a focus on the large number of repetitions, if feasible, seems to lead to less variable results, even with a lower nominal volume.

Two additional observations merit discussion. First, the rCBF estimated by FAIR was significantly higher compared with PICORE. The rCBF calculation was performed with standard parameters by using commercially available software directly inline on the MR imaging scanner. Note that despite the highly significant correlation between the values obtained with these ASL sequences, there is still a systematic offset difference between them. Correspondingly, previous investigations comparing ASL and $\mathrm{H}_{2}\left[{ }^{15} 0\right]$-PET (water PET) as a reference standard ${ }^{22,23}$ as well as combined ASL and PET imaging ${ }^{24,25}$ also showed good correlations between ASL and water PET. The correlation coefficients are, however, unequal to 1. Likewise, another study compared 2D-versus-3D pseudocontinuous ASL and $\mathrm{H}_{2}\left[{ }^{15} 0\right]$-PET in 6 healthy controls, 6 subjects with MCI, and 1 patient with Alzheimer disease and again found a systematic offset between 2D-versus-3D ASL and between ASL-versus-water PET. ${ }^{26}$ Moreover, the placement of the imaging slab significantly changed the rCBF estimation in ASL and the correlation with water PET, ${ }^{27}$ indicating the need for rigorous standardization of this parameter. In posterior circulation. There were, however, certain exceptions to this rule. PICORE provided higher signal in the bilateral head of caudate nucleus and posterior insula, both part of the anterior circulation, while FAIR provided higher signal in the occipital pole and the posterior division of the cingulate gyrus, both part of the posterior circulation. Along the same lines, a previous investigation assessed the effect of imaging slab positioning and arterial transit time of ASL compared with $\mathrm{H}_{2}\left[{ }^{15} 0\right]-\mathrm{PET}^{27}$ The spatial distribution of the direct comparison between ASL and water PET partly overlaps the patterns of the current investigation, indicating that systematic ASL sequencerelated differences in arterial transit time might contribute to the observed spatial differences between FAIR and PICORE. The exact origin of these regional differences between both ASL sequences, irrespective of the vascular territory, remains unclear. However, one of the differences between both ASL sequences was the use of a reference image for FAIR yet not for PICORE, which might lead to subtle differences between both sequences based on the level of T1-weighting between them.

Regarding the selective difference of perfusion in the posterior circulation, superselective or territorial ASL allows labeling of only selected vessels of the neck and demonstrated that, for example, the vascular supply of deep cerebral structures largely varies depending on the presence of the anatomic variant of a fetal configuration of the posterior cerebral artery. ${ }^{29,30}$ However, if anatomic variants are present within an individual, these equally affect both ASL sequences in our investigation. Consequently, such anatomic variants may explain regional ASL variations between individuals yet not between ASL sequences within the same indi- 

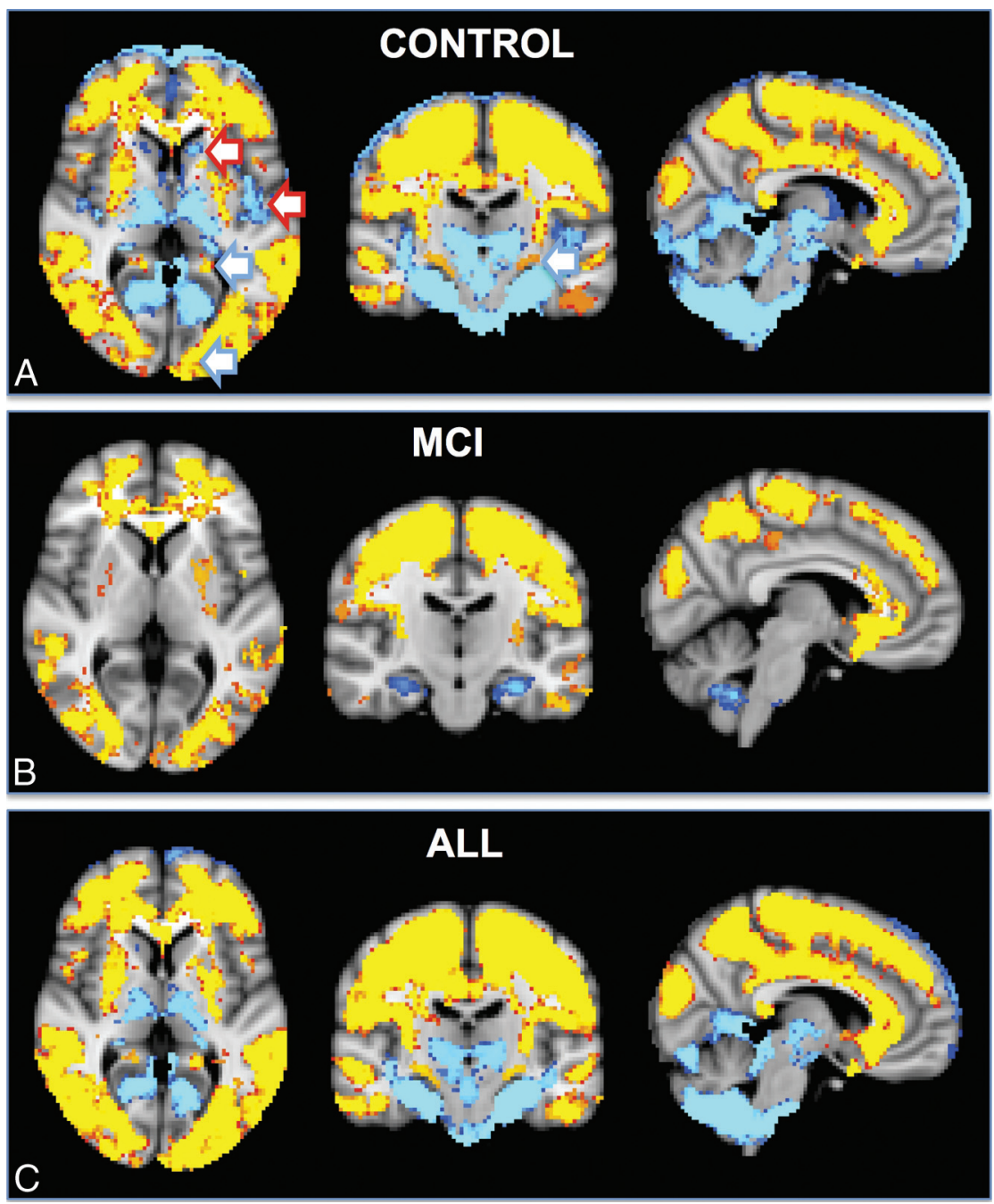

FIG 4. The spatial distribution of the differences between FAIR and PICORE ASL. Higher signal for FAIR (red-yellow) was present notably in both convexities, which largely but not completely respected the anterior circulation, for example posterior division of the cingulate gyrus (posterior cerebral artery, posterior circulation). In contrast, PICORE had higher signal in the bilateral thalami and occipital and infratentorial regions, largely but not completely respecting the posterior circulation and additionally in the bilateral head of caudate nucleus (lenticulostriate arteries, anterior circulation) and posterior insula (middle cerebral artery, anterior circulation).

vidual. A potential subtle difference in ASL imaging could also be related to the direction of the applied QUIPSS II/Q2TIPS saturation pulses. In the current investigation, these pulses were always parallel to the acquisition (and labeling) slabs, excluding a potential bias between both ASL sequences.

Most interesting, although PICORE had globally lower signal than FAIR, this was not the case in the bilateral mesial temporal regions. Mesial temporal atrophy is a well-known hallmark of Alzheimer disease, ${ }^{31}$ yet previous ASL studies did not reliably identify local hypoperfusion in this area. ${ }^{2-5}$ The observation that PICORE provides higher signal in this region indicates that this ASL sequence might be tuned to be particularly sensitive in regions prone to neurodegeneration.

\section{Limitations}

Both ASL sequences had different parameters, including spatial resolution (similar pixel size but different section thickness and partial Fourier factor) because they were individually optimized according to the recommended standards and options available on our MR imaging scanner. Note that both ASL sequences used parameters that were, at the date of the study start, in agreement with the most recent guidelines. ${ }^{16}$ As mentioned above, in clinical settings, these variations are likely much more important when using different MR imaging scanners, head coils, and so forth, highlighting the need to calibrate each ASL sequence.

\section{CONCLUSIONS}

Overall, a large number of repetitions in our FAIR sequence had lower interindividual variability than the smaller number of repetitions in our PICORE sequence. The current implementation of FAIR is therefore recommended over the current implementation of PICORE for imaging in neurodegeneration. In more detail, the regional differences and global offset between both ASL sequences highlight the need to calibrate ASL sequences. Moreover, these results indicate that dedicated ASL sequences might be developed to assess specific target regions of the brain.

\section{ACKNOWLEDGMENTS}

We thank all volunteers and patients for participating in our study. We also thank Andreas J. Bartsch and Joseph Pfeuffer for their constructive help during the manuscript preparation.

Disclosures: Cristelle Rodriguez, Simona TomaRELATED: Grant: Spezialprogramm Universitäre Medizin (SPUM) 33CM30_124111/1,* FNS 320030_ 129690/1, ${ }^{*}$ Comments: the conversion of mild cognitive impairment to Alzheimer's disease; and early prediction of cognitive decline in very old individuals: combining disease-related, structural and functional markers. Xavier Golay—RELATED: Grant: European Cooperation in Science and Technology (COST) Agency, ${ }^{*}$ Comments: Grant "BM1103: ASL in Dementia" is a cooperation grant throughout European Union countries, of which I am the Principal Investigator. Panteleimon Giannakopoulos-RELATED: Grant: SPUM 33CM30_124111/1, * FNS 320030_129690/1, “ Comments: the conversion of mild cognitive impairment to Alzheimer's disease; and Early prediction of cognitive decline in very old individuals: combining disease-related, structural and functional markers; UNRELATED: Expert Testimony: witness reports for forensic psychiatry; Grants/Grants Pending: Swiss National Foundation for Research, ${ }^{\star}$ Comments: fees received by the University Hospitals of Geneva for clinical research (local private foundations which support pilot projects with small amounts of money); Payment for Lectures (including service on Speakers Bureaus): University of Lausanne, * Fondation Leenaards, Lausanne, * pensions for old age psychiatry, ${ }^{\star}$ Comments: honoraria for conferences (usually less than US \$1000). Sven Haller-RELATED: Grant: Swiss National Science Foundation, ${ }^{\star}$ Comments: grant supporting the basic project; UNRELATED: Grants/Grants Pending: Swiss National Science Foundation*; Payment for Lectures (including service on Speakers Bureaus): Various other sources. * Money paid to the institution.

\section{REFERENCES}

1. Roberts DA, Detre JA, Bolinger L, et al. Quantitative magnetic resonance imaging of human brain perfusion at $1.5 \mathrm{~T}$ using steady-state inversion of arterial water. Proc Natl Acad Sci U S A 1994;91:33-37

2. Yoshiura T, Hiwatashi A, Noguchi T, et al. Arterial spin labelling at 
3-T MR imaging for detection of individuals with Alzheimer's disease. Eur Radiol 2009;19:819-25

3. Yoshiura T, Hiwatashi A, Yamashita K, et al. Simultaneous measurement of arterial transit time, arterial blood volume, and cerebral blood flow using arterial spin-labeling in patients with Alzheimer disease. AJNR Am J Neuroradiol 2009;30:1388-93

4. Dai W, Lopez OL, Carmichael OT, et al. Mild cognitive impairment and Alzheimer disease: patterns of altered cerebral blood flow at MR imaging. Radiology 2009;250:856-66

5. Binnewijzend MA, Kuijer JP, Benedictus MR, et al. Cerebral blood flow measured with 3D pseudocontinuous arterial spin-labeling MR imaging in Alzheimer disease and mild cognitive impairment: a marker for disease severity. Radiology 2013;267:221-30

6. Wolk DA, Detre JA. Arterial spin labeling MRI: an emerging biomarker for Alzheimer's disease and other neurodegenerative conditions. Curr Opin Neurol 2012;25:421-48

7. Xekardaki A, Rodriguez C, Montandon ML, et al. Arterial spin labeling may contribute to the prediction of cognitive deterioration in healthy elderly individuals. Radiology 2015;274:490-99

8. Bozoki AC, Korolev IO, Davis NC, et al. Disruption of limbic white matter pathways in mild cognitive impairment and Alzheimer's disease: a DTI/FDG-PET study. Hum Brain Mapp 2012;33:1792-802

9. Pagani M, Dessi B, Morbelli S, et al. MCI patients declining and not-declining at mid-term follow-up: FDG-PET findings. Curr Alzheimer Res 2010;7:287-94

10. Herholz K, Salmon E, Perani D, et al. Discrimination between Alzheimer dementia and controls by automated analysis of multicenter FDG PET. Neuroimage 2002;17:302-16

11. Du AT, Jahng GH, Hayasaka S, et al. Hypoperfusion in frontotemporal dementia and Alzheimer disease by arterial spin labeling MRI. Neurology 2006;67:1215-20

12. Buxton RB, Frank LR. A model for the coupling between cerebral blood flow and oxygen metabolism during neural stimulation. J Cereb Blood Flow Metab 1997;17:64-72

13. Aubert A, Costalat R. A model of the coupling between brain electrical activity, metabolism, and hemodynamics: application to the interpretation of functional neuroimaging. Neuroimage 2002;17: 1162-81

14. Kim SG. Quantification of relative cerebral blood flow change by flow-sensitive alternating inversion recovery (FAIR) technique: application to functional mapping. Magn Reson Med 1995;34:293-301

15. Wong EC, Buxton RB, Frank LR. Implementation of quantitative perfusion imaging techniques for functional brain mapping using pulsed arterial spin labeling. NMR Biomed 1997;10:237-49

16. Alsop DC, Detre JA, Golay X, et al. Recommended implementation of arterial spin-labeled perfusion MRI for clinical applications: a consensus of the ISMRM perfusion study group and the European consortium for ASL in dementia. Magn Reson Med 2014 Apr 8. [Epub ahead of print]

17. Folstein MF, Folstein SE, McHugh PR. "Mini-mental state": a practical method for grading the cognitive state of patients for the clinician. J Psychiatr Res 1975;12:189-98
18. Luh WM, Wong EC, Bandettini PA, et al. QUIPSS II with thin-slice TI1 periodic saturation: a method for improving accuracy of quantitative perfusion imaging using pulsed arterial spin labeling. Magn Reson Med 1999;41:1246-54

19. Wang J, Licht DJ, Jahng GH, et al. Pediatric perfusion imaging using pulsed arterial spin labeling. J Magn Reson Imaging 2003;18:404-13

20. Fazekas F, Chawluk JB, Alavi A, et al. MR signal abnormalities at $\mathbf{1 . 5}$ $\mathrm{T}$ in Alzheimer's dementia and normal aging. AJR Am J Roentgenol 1987;149:351-56

21. Smith SM, Nichols TE. Threshold-free cluster enhancement: addressing problems of smoothing, threshold dependence and localisation in cluster inference. Neuroimage 2009;44:83-98

22. Heijtel DF, Mutsaerts HJ, Bakker E, et al. Accuracy and precision of pseudo-continuous arterial spin labeling perfusion during baseline and hypercapnia: a head-to-head comparison with (1)(5)O $\mathrm{H}(2) \mathrm{O}$ positron emission tomography. Neuroimage 2014;92:182-92

23. Bokkers RP, Bremmer JP, van Berckel BN, et al. Arterial spin labeling perfusion MRI at multiple delay times: a correlative study with $\mathrm{H}(2)(15) \mathrm{O}$ positron emission tomography in patients with symptomatic carotid artery occlusion. J Cereb Blood Flow Metab 2010; 30:222-29

24. Zhang K, Herzog H, Mauler J, et al. Comparison of cerebral blood flow acquired by simultaneous $[15 \mathrm{O}]$ water positron emission tomography and arterial spin labeling magnetic resonance imaging. J Cereb Blood Flow Metab 2014;34:1373-80

25. Stegger L, Martirosian P, Schwenzer N, et al. Simultaneous PET/MR imaging of the brain: feasibility of cerebral blood flow measurements with FAIR-TrueFISP arterial spin labeling MRI. Acta Radiol 2012;53:1066-72

26. Kilroy E, Apostolova L, Liu C, et al. Reliability of two-dimensional and three-dimensional pseudo-continuous arterial spin labeling perfusion MRI in elderly populations: comparison with 150 -water positron emission tomography. J Magn Reson Imaging 2014;39: 931-39

27. Qiu M, Paul Maguire R, Arora J, et al. Arterial transit time effects in pulsed arterial spin labeling CBF mapping: insight from a PET and MR study in normal human subjects. Magn Reson Med 2010;63: 374-84

28. Wang Y, Saykin AJ, Pfeuffer J, et al. Regional reproducibility of pulsed arterial spin labeling perfusion imaging at $3 \mathrm{~T}$. Neuroimage 2011;54:1188-95

29. Hendrikse J, Petersen ET, Chng SM, et al. Distribution of cerebral blood flow in the nucleus caudatus, nucleus lentiformis, and thalamus: a study of territorial arterial spin-labeling MR imaging. Radiology 2010;254:867-75

30. Hartkamp NS, Petersen ET, De Vis JB, et al. Mapping of cerebral perfusion territories using territorial arterial spin labeling: techniques and clinical application. NMR Biomed 2013;26:901-12

31. Fox NC, Warrington EK, Freeborough PA, et al. Presymptomatic hippocampal atrophy in Alzheimer's disease: a longitudinal MRI study. Brain 1996;119:2001-07 